\title{
Delayed Diagnosis of Multibacillary Leprosy: A Report of Eight Cases
}

\author{
Maria Angela Bianconcini Trindade ${ }^{1,2}$, Tatiana Cristina Nogueira Varella ${ }^{1}$, Claudia Gertrudis Cardoza Cisneros ${ }^{1}$, Vanessa \\ Bottini and Ana Karina Alves Moura ${ }^{1}$ \\ ${ }^{1}$ Dermatology Clinic, Clinical Hospital of São Paulo University; ${ }^{2}$ Health Institute, Health State Secretary; São Paulo, SP, Brazil
}

Leprosy is an important public health problem in Brazil. However, this disease is still poorly diagnosed in its early stages, leading to permanent disability and disfigurement. We examined eight patients with clinical and histological diagnosis of multibacillary leprosy who were being treated for other diseases for about three years without clinical hypothesis of leprosy. These cases illustrate the importance of medical education and public information about leprosy's signs and symptoms for prompt recognition and treatment, which are necessary to prevent permanent disabilities and eradicate the disease.

Key-Words: Misdiagnosis, late diagnosis, multibacillary leprosy.

Leprosy is an important public health problem in endemic areas. In spite of governmental strategies for reducing the leprosy burden and leprosy control, with declining incidence since 1991 due to new strategies for dealing with this disease, including multidrug therapy with reduced duration of treatment and the adoption of new cure criteria, more than 250,000 new cases were registered in 2007, including about 40,000 in Brazil and 140,000 in India [1,2].

Leprosy is a chronic disease caused by an intracellular bacillus, Mycobacterium leprae, which multiplies very slowly. The incubation period of this disease is about five years. It is transmitted via secretion droplets, from the nose and mouth, during close and frequent contacts with untreated patients, which are the most important transmission foci. Clinically, leprosy mainly affects the nerves and skin. The commonest skin lesions are erythematous, hypopigmented and infiltrated plaques, which have reduced sensibility. Damage to the nerves explains the sensory loss; if untreated, there can be progressive and permanent disabilities. Other clinical aspects of leprosy are reactions, which may be defined as acute phases within the usual chronic evolution of the disease; sometimes these define the moment of diagnosis [3-5]. Diagnosis is clinical and treatment is made at public health facilities [2].

Eight patients were evaluated at the Clinical Dermatology Division of the Hospital das Clínicas da Universidade de São Paulo, where diagnosis of multibacillary leprosy was made clinically and histologically. All eight patients had been initially misdiagnosed. We present these cases, followed by a discussion regarding various aspects of Hansen disease and the importance of early diagnosis for preventing complications and transmission (Table 1).

Received on 6 September 2008; revised 19 January 2009.

Address for correspondence: Dr. Maria Angela Bianconcini Trindade. Rua Cristiano Viana 450 Ap 163, Jardim América. Zip code: 05411000, São Paulo-SP, Brasil. Phone/fax: 551131598279. E-mail: angelatrindade@uol.com.br.

The Brazilian Journal of Infectious Diseases 2009;13(1):155-157. (C) 2009 by The Brazilian Journal of Infectious Diseases and Contexto Publishing. All rights reserved.

\section{Discussion}

Because of the long incubation period, infection with Mycobacterium leprae may not be evident in its early stages. This peculiarity can lead to misdiagnosis and consequently to long-term morbidity and disfigurement. The ability to recognize this disease and provide prompt therapy is essential to prevent disfiguring and other undesirable consequences [6,7].

Leprosy's diagnosis is clinical; it is based on the finding of cutaneous lesions with altered sensation and thickened peripheral nerves. This disease has several clinical presentations, which vary from well delimited and clearly recognizable plaques to poorly defined macules, anesthetized areas, alopecia, dryness and diffuse infiltration of the skin. These clinically ill-defined lesions can be easily misdiagnosed, even when patients are examined by experts, leading to late diagnosis, which increases the transmission period and the probability of disabilities. Consequently, patient history and physical examination are fundamental steps in leprosy investigation. If investigated, the finding of incipient clinical signs can lead to earlier diagnosis. Since early detection and treatment is the best way to eliminate leprosy, it is clear that in Brazil, which is an endemic country, these ill-defined precocious symptoms need to be fully and extensively investigated [8-10].

Our patients presented to health services with skin lesions, anesthetized areas, amyotrophy and various other polymorphic signs and symptoms of chronic leprosy; but they were misdiagnosed by doctors of different specialties (gynecologist, infectologist, general clinicians, dermatologist, otorrinolaryngologist, plastic surgeon, rheumatologist, and vascular surgeon). Even when they had leprosy-provoked disabilities the patients were treated for other conditions. It usually took at least three years for a correct diagnosis; during this time, most of them developed complications due to late diagnosis of this disease.

We concluded that it is very important to teach both the public and health workers about leprosy. Familiarity with this disease is essential to initiate appropriate therapy and avert lasting sequels. Early case detection and treatment with multidrug therapy remains the cornerstone of leprosy control. An integrated approach that uses informed health-care workers and easily accessible leprosy services near patients' 
금

$\Xi$

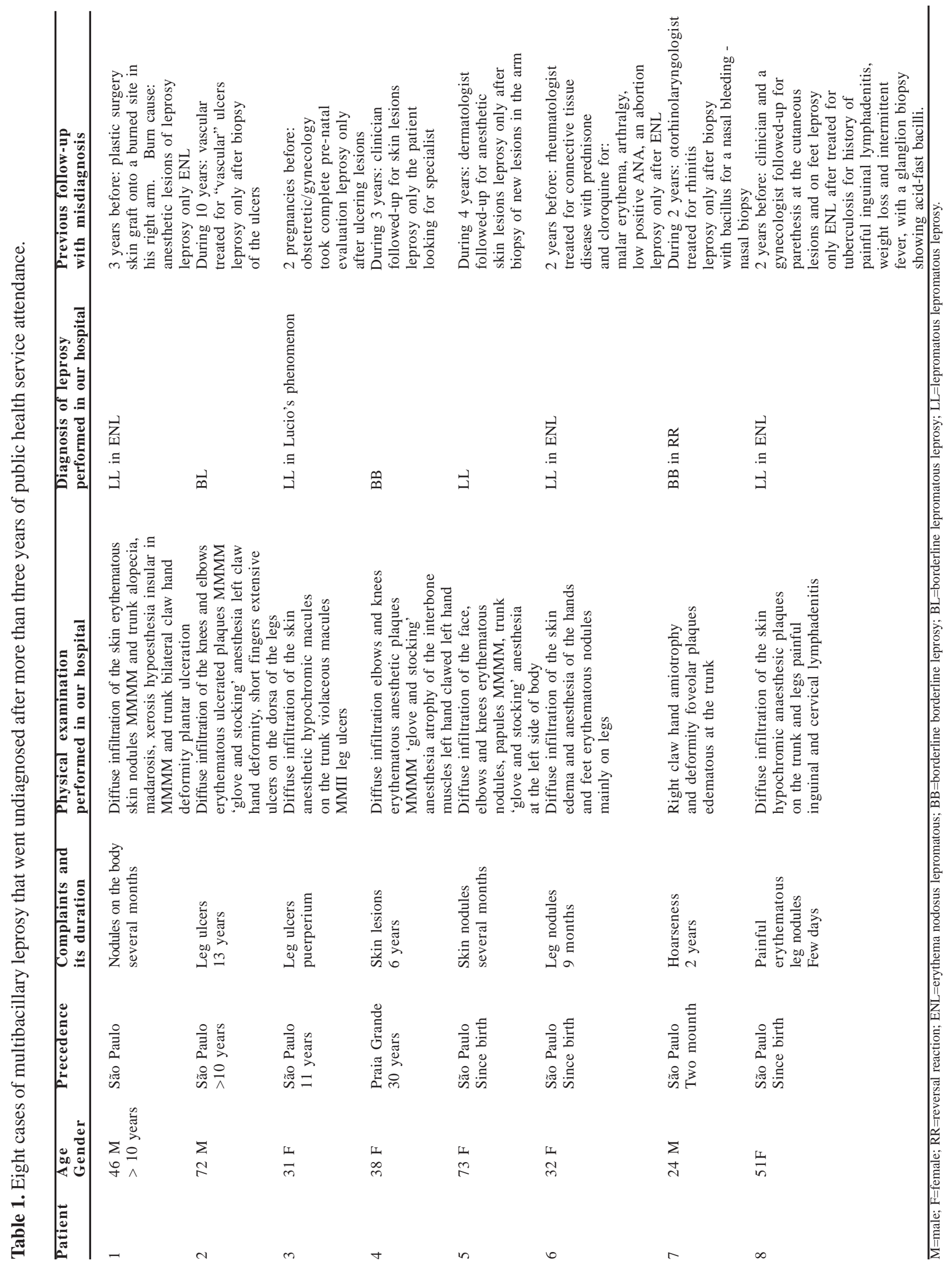


homes and access to tertiary health-care services will continue to be the key strategies to achieve worldwide decline of this disease and of its consequences [9].

\section{Acknowledgments}

We thank the leprosy patients for teaching us about patience.

\section{References}

1. World Health Organization. Global Leprosy Situation, beginning of 2008. Wkly Epidemiol Rec 2008;33(83):293-300.

2. Global Strategy for further reducing the leprosy burden and sustaining leprosy control activities (plan period 2005-2010). www.who.int/entity/lep/resources/Global Strategy.pdf. Acessed in 04.03.2008

3. Walker SL, Lockwood DNJ. Leprosy. Clinics Dermatology 2007;25:165-72.
4. Souza C.S. Hanseníase: Formas clínicas e diagnóstico diferencial. Medicina, Ribeirão Preto 1997;30:325-34.

5. Naafs B. Current views on reactions in leprosy. Indian J Lepr 2000;72:97-12.

6. Hartzel J.D., Michael Zapor L.T.C., Suzette Peng S., Timothy Straight M.A. Leprosy: A Case Series and Review. Southern Medical Journal 2004;97(12):1252-6.

7. Ladhani S.B.S. Leprosy disabilities: the impact of multidrug therapy (MDT). International Journal of Dermatology 1997,36(8):561-72.

8. Trindade M.A.B., Nemes M.I.B. Incapacidades físicas em Hanseníase no momento do diagnóstico: características epidemiológicas dos casos registrados de 1983 a 1988 no Estado de São Paulo. Hansen Int 1992;17:8-14.

9. Trindade M.A., Manini M.I., Lastória J.C. Role of dermatologists in leprosy elimination and in the post-elimination era. Lepr Rev 2007;78(1):50-1.

10. Monot M., Honoré N., Garnier T., Araoz R., et al. On the origin of leprosy. Science 2005;308(5724):1040-2. 